at admission were independently associated with differences in survival.

\section{PS-044 NEONATAL UNIT ADMISSION VOLUME IMPACTS IN-HOSPITAL MORTALITY FOR VERY PRETERM INFANTS IN EUROPE: RESULTS FROM THE EPICE COHORT}

'E Lebreton, 'B Blondel, ${ }^{2} \mathrm{~J}$ Gadzinowski, 'M Bonet, ' Pediatric Epidemiology Research Team Center for Epidemiology and Biostatistics (U1153), Inserm, Paris, France; ${ }^{2}$ Department of Neonatology, Poznan University of Medical Sciences, Poznan, Poland

10.1136/archdischild-2014-307384.341

Background and aims Studies have shown that very preterm infants (VPTI) have higher survival when they are born in a maternity unit associated with a high volume neonatal unit. We sought to analyse the impact of nursery volume on in-hospital mortality in Europe.

Methods Data come from the EPICE (Effective Perinatal Intensive Care in Europe) project, a population-based study of VPTI born in 19 European regions over 12 months in 2012-2013. We included all live births between 24 and 31 weeks of gestation without severe congenital anomalies $(\mathrm{n}=7383)$ born in 350 maternity units. Volume was defined as the number of observed admissions to the neonatal unit associated with the delivery hospital. Our outcome was death before discharge home. We assessed the impact of volume, analysed as a continuous variable, using multi-level logistic regression and considering case-mix (gestational age, sex, small for gestational age, multiple pregnancy, maternal age and parity).

Results $8 \%$ of VPTI were born in maternity units with less than 10 neonatal admissions, $8 \%$ in units with 10 to 29 admissions, $11 \%$ in units with 30 to 49 admissions, $42 \%$ in units with 50 to 99 admissions and $31 \%$ in units with $\geq 100$ admissions. After adjustment, we found a significant linear association between volume and in-hospital mortality, with an odds ratio of 0.95 (0.91-0.98) for 20 additional admissions.

Conclusions VPTI born in maternity units associated with high volume neonatal units had better survival. Delivery in matenity units with larger neonatal units may contribute to improved outcomes in this population.

\section{PS-045 EXPLAINING THE DIFFERENCES IN MORTALITY RATES FOR VERY PRETERM BIRTHS ACROSS EUROPE: THE EPICE STUDY}

${ }^{1} \mathrm{ES}$ Draper, ${ }^{1} \mathrm{BN}$ Manktelow, ${ }^{2} \mathrm{M}$ Bonet, ${ }^{2} \mathrm{~J}$ Zeitlin, ${ }^{3} \mathrm{EG}$ Epice Group. ${ }^{1}$ Health Sciences, College of Medecine Biological Sciences and Psychology, Leicester, UK; ${ }^{2}$ U1153 - Equipe Epopé, INSERM, Paris, France; ${ }^{3}$ INSERM U1153 - Equipe Epopé, FP7 Project Group Author, Paris, France

\subsection{6/archdischild-2014-307384.342}

Background Mortality rates for very preterm births (VPTBs) show wide variation across Europe. Some, of this variation can be explained by a lack of standardised data collection and reporting. Using the standardised EPICE population-based cohort of VPTBs we investigate the potential explanatory factors for the variation in the in-hospital mortality rates between 19 European study regions.

Methods All births between $22^{+0}$ and $31^{+6}$ weeks of gestational age were included in the EPICE birth cohorts in 19 regions in
11 European countries. A standardised data collection system was established in each of the regions; ascertainment was validated against birth registers. All VPTBs were followed to death or discharge home from neonatal care. Mortality rates were calculated for the total cohort $(\sim 10,000)$, live born infants and those admitted for neonatal care. Assessment of the potential maternal and infant explanatory factors for the variations in standardised mortality rates were investigated using multilevel logistic regression.

Results Crude in-hospital mortality rates for (i) total very preterm birth cohort $22^{+0}$ to $31^{+6}$ weeks gestation (excluding TOPs for congenital anomaly), ranged from $19.5 \%$ to $48.9 \%$ by region; (ii) all live births: $6.7-20.9 \%$ and (iii) for admissions to neonatal care: 4.9-18.3\%. Following adjustment for maternal and infant characteristics the variation in these rates reduced to: total cohort 23.5-39.3\%; live births $10.2-17.7 \%$ and NIC admissions $7.5-15.2 \%$.

Conclusions Only a small proportion of the variation in the standardised mortality rates was explained by the maternal and infant characteristics. Further work will investigate variation in the timing of death.

\section{PS-046 FIRST DAY HEART RATE CHARACTERISTICS PREDICT DEATH AND ADVERSE EVENTS IN PRETERM INFANTS}

${ }^{1} \mathrm{~K}$ Fairchild, ${ }^{1} \mathrm{BA}$ Sullivan, ${ }^{2} \mathrm{CJ}$ McClure, ${ }^{1} \mathrm{~J}$ Hicks, ${ }^{3} \mathrm{DE}$ Lake, ${ }^{3} \mathrm{JR}$ Moorman. ${ }^{1}$ Pediatrics, University of Virginia, Charlottesville, USA; ${ }^{2}$ School of Medicine, University of Virginia, Charlottesville, USA; ${ }^{3}$ Medicine, University of Virginia, Charlottesville, USA

\subsection{6/archdischild-2014-307384.343}

Purpose Abnormal heart rate characteristics (HRC) of decreased variability and decelerations occur in preterm infants with sepsis and other pathologic conditions. We sought to determine whether an HRC index (HeRO score) in the first day after birth predicts death and morbidities and to compare it to an established risk index, the Score for Neonatal Acute Physiology (SNAP-II).

Methods The HRC index was analysed within $24 \mathrm{~h}$ of birth in 163 extremely low birth weight infants, and SNAP-II was calculated when data were available. Associations between the maximum HRC index (HRC-1), SNAP-II, and death and major morbidities were analysed using logistic regression to correct for gestational age.

Results HRC-1 was significantly associated with death, severe head ultrasound abnormalities (sHUS = grade 3-4 intraventricular haemorrhage or cystic periventricular leukomalacia), and lateonset septicemia (LOS) (Table). SNAP-II could be calculated in 122 cases $(75 \%)$ and was correlated with HRC-1 $(\mathrm{r}=0.50, \mathrm{p}<$ $0.0001)$ and with death, sHUS, and bronchopulmonary dysplasia

\begin{tabular}{llll}
\multicolumn{4}{l}{ Abstract PS-046 Table 1 } \\
\hline Outcome & $\%$ with Outcome & HRC-1 $\mathbf{p ~ = ~ *}$ & SNAP $\mathbf{p ~ = ~ * ~}$ \\
\hline Death & $19 \%$ & 0.009 & $<0.001$ \\
Severe HUS & $19 \%$ & 0.006 & 0.001 \\
Late-onset septicemia & $24 \%$ & 0.049 & 0.714 \\
NEC/SIP & $13 \%$ & 0.565 & 0.142 \\
BPD & $55 \%$ & 0.186 & 0.018 \\
Severe ROP & $8 \%$ & 0.555 & 0.204 \\
Survival, No Morbidities & $25 \%$ & 0.029 & 0.010 \\
* corrected for gestational age & & \\
\hline
\end{tabular}


(BPD). Neither score predicted necrotizing enterocolitis or spontaneous intestinal perforation (NEC/SIP), or severe retinopathy of prematurity (ROP).

Conclusion The HRC index in the first day after birth predicts death and multiple morbidities in ELBW infants and is strongly correlated with SNAP-II, an established illness severity score.

\section{PS-047 TRADEOFFS BETWEEN MORTALITY AND MORBIDITY FOR VERY PRETERM INFANTS: RESULTS FROM THE EPICE COHORT}

J Zeitlin', AK Edstedt Bonamy², M Bonet', ES Draper ${ }^{3}$, E Epice Research Group ${ }^{4}$. 'Obstetrical Perinatal and Pediatric Epidemiology Research Team Center for Epidemiology and Biostatistics (U1153), Inserm, Paris, France; 'Department of Women's and Children's Health, Karolinska Institutet, Stockholm, Sweden; ${ }^{3}$ Department of Health Science, University of Leicester, Leicester, UK; ${ }^{4}$ Group Author, European Project, Paris, France

\subsection{6/archdischild-2014-307384.344}

Background and aims There is concern that declines in mortality after very preterm birth are accompanied by higher morbidity as more fragile babies survive. We investigated whether rates of severe neonatal morbidity were higher in regions with low versus high mortality.

Methods The EPICE cohort included all births between $22+0$ and $31+6$ weeks of gestation in 19 European regions in 20112012 ( $\mathrm{N}=7878$ live births). We divided the cohort into tertiles based on regional in-hospital mortality rates excluding deaths due to congenital anomalies: low $(<12.0 \%)$, medium $(12.0$ to $14.9 \%)$ and high $(\geq 15.0 \%)$. Severe neonatal morbidity was defined as intraventricular haemorrhage grades III-IV, cystic periventricular leukomalacia, surgery/peritoneal drainage for necrotizing enterocolitis or retinopathy of prematurity (grades $\geq 3$ ). Bronchopulmonary dysplasia (BPD) was analysed separately. Multilevel logistic regressions were used to model regional-level effects.

Results 6770 infants were discharged home. 12.2\% had at least one severe morbidity (regional range: $3.8 \%$ to $24.4 \%$ ) and $15.3 \%$ had BPD (regional range: $6.7 \%$ to $34.9 \%$ ). Rates of severe morbidity were $11.7 \%, 12.1 \%$ and $12.9 \%$ in low, medium and high mortality regions, respectively $(\mathrm{p}=0.45)$. BPD was higher in low mortality regions $(18.7 \%, 16.7 \%$ and $10.2 \%$, respectively $(\mathrm{p}<0.001)$. The odds ratio for severe morbidity associated with birth in a low versus high mortality region was 0.83 (95\% CI: 0.55-1.25) and for BPD was 1.81 (95\% CI: 1.08-3.0).

Conclusion Rates of severe neonatal morbidity and BPD vary greatly between regions in Europe. BPD rates were higher in lower mortality regions, but this was not true for other morbidities.

\section{PS-048 TRENDS IN SNAPPE-II AND CRIB II SCORES OVER A 15-YEAR PERIOD}

MC de VoS, EJH Mulder, J Schuurmans, D Kwakkel, JB Derks, F Groenendaal. Perinatal Center, Wilhelmina Children's Hospital University Medical Center Utrecht, Utrecht, Netherlands

\subsection{6/archdischild-2014-307384.345}

Background During the last decades mortality of preterm born neonates has declined in the Western world.
Aim We hypothesised that the decrease in mortality in very preterm neonates over a period of 15 years was associated with a decrease in illness severity scores (SNAPPE-II and CRIB II).

Subjects/methods Inborn neonates (January 1997 until December $2011 ; \mathrm{n}=928$ ) with a gestational age of 26.0-29.9 weeks and without congenital malformations.

SNAPPE-II and CRIB II scores, mortality ( $<120$ days $)$ and morbidity (severe intraventricular haemorrhage (IVH), severe cystic periventricular leukomalacia (cPVL), bronchopulmonary dysplasia (BPD), perforated necrotizing enterocolitis (NEC) and severe retinopathy of prematurity (ROP)) were recorded. Based on important changes in care, subjects were categorised into three periods of year of birth (1: 1997-1999, 2: 2000-2005, and 3: 2006-2011) and outcomes were analysed using multivariable analysis including SNAPPE-II/CRIB II scores, period, gestational age, gender, and their interactions.

Results SNAPPE-II and CRIB II scores were significantly lower for gestational ages between 26.0 and 29.9 in period 3 versus period 1 ( $\mathrm{p}=0.002 ; \mathrm{p}=0.018$, respectively) in a cubic model analysis. The risks of mortality and serious morbidity were significantly lower for similar SNAPPE-II and CRIB II scores in period 3 versus period 1 ( $\mathrm{p}=0.010 ; \mathrm{p}=0.041$, respectively). Females had a significantly lower risk of mortality and serious morbidity than males ( $\mathrm{p}=0.031 ; \mathrm{p}<0.0001$, respectively).

Conclusions SNAPPE-II and CRIB II scores decreased over a 15 -year period. Meanwhile, the risk of mortality and serious morbidity for similar SNAPPE-II and CRIB II scores decreased suggesting substantial improvement in perinatal care.

\section{PS-049 QUALITY APPRAISAL OF INTERNATIONAL GUIDELINES ON PERINATAL CARE OF EXTREMELY PREMATURE INFANTS}

${ }^{1} \mathrm{~N}$ Binepal, ${ }^{2} \mathrm{G}$ Moore, ${ }^{2} \mathrm{~B}$ Lemyre, ${ }^{2} \mathrm{~T}$ Daboval, ${ }^{3} \mathrm{~S}$ Dunn, ${ }^{4} \mathrm{~S}$ Leduc. ${ }^{1}$ Pediatrics, Children's Hospital of Eastern Ontario, Ottawa, Canada; ${ }^{2}$ Neonatology, Children's Hospital of Eastern Ontario, Ottawa, Canada; ${ }^{3}$ Children's Hospital of Eastern Ontario Research Institute, Children's Hospital of Eastern Ontario, Ottawa, Canada; ${ }^{4}$ Life Sciences, University of Ottawa, Ottawa, Canada

\subsection{6/archdischild-2014-307384.346}

Background Clinicians often refer to published or local guidelines when counselling expectant parents on perinatal care decisions at the limits of viability. The number and quality of guidelines used worldwide is unknown.

Objectives Assess quality of international guidelines regarding perinatal care of 22-25 wk GA infants.

Methods MEDLINE, Pre-MEDLINE and TRIP databases were searched for international guidelines using specific criteria. Titles/abstracts were screened and the final selected guidelines were reviewed and appraised by two reviewers using the validated AGREE-II (Appraisal of Guidelines for Research and Evaluation Instrument) tool. This instrument assesses guideline quality (scope, stakeholder involvement, rigour/transparency, clarity, applicability, editorial independence). Items with score differences $>3$ were reviewed for discussion.

Results Database and grey search yielded 263 publications. Screening left 37 guidelines, 16 of which met all inclusion criteria. Regions represented included North America, Australia and Europe. Appraisal using the AGREE-II tool by three independent appraisers revealed deficits within all domains, predominantly 'rigour of development' and 'applicability'. Overall quality scores 